\section{A quantitative real time- polymerase chain reaction approach for estimating processed animal proteins in feed: preliminary data}

\section{Daniela Marchis, Alessandro Benedetto, Giuseppina Amato, Beatrice Brusa, \\ Stefania Squadrone, \\ Maria Cesarina Abete}

Istituto Zooprofilattico Sperimentale del Piemonte, Liguria e Valle D'Aosta, Torino, Italy

\begin{abstract}
Lifting of the ban on the use of processed animal proteins (PAPs) from non-ruminants in non-ruminant feed is in the wind, avoiding intraspecies recycling. Discrimination of species will be performed through polymerase chain reaction (PCR), which is at a moment a merely qualitative method. Nevertheless, quantification of PAPs in feed is needed. The aim of this study was to approach the quantitative determination of PAPs in feed through Real Time (RT)-PCR technique; three different protocols picked up from the literature were tested. Three different kind of matrices were examined: pure animal meals (bovine, chicken and pork); one feed sample certified by the European reference laboratory on animal proteins (EURL AP) in feed spiked with $0.1 \%$ bovine meal; and genomic DNAs from bovine, chicken and pork muscles. The limit of detection (LOD) of the three protocols was set up. All the results obtained from the three protocols considered failed in the quantification process, most likely due to the uncertain copy numbers of the analytical targets chosen. This preliminary study will allow us to address further investigations, with the purpose of developing a RT-PCR quantitative method.
\end{abstract}

\section{Introduzione}

La decima revisione della seconda edizione di TSE roadmap II: a strategy paper on transmissible spongiform encephalopathies for 2010-2015 (Commissione Europea, 2010) riporta come uno degli obiettivi strategici la revisione del feed ban così come previsto ad oggi dalla legislazione europea, sollecitando la reintroduzione delle proteine animali trasformate (PAT) nell'alimentazione dei non-ruminanti. L'utilizzo delle PAT deve necessariamente evitare il riciclo intraspecie. La PCR, identificata dallo European reference laboratory
(EURL) on animal proteins (AP) come la metodica di scelta per l'identificazione di specie, fornisce un risultato di tipo qualitativo (presenza/assenza). L'impossibilità di distinguere tra cross-contaminazioni e possibili frodi commerciali in realtà costituisce un problema di grande rilevanza. In questo studio è stata esplorata la possibilità di utilizzare la real time-polymerase chain reaction (RT-PCR) per la determinazione quantitativa delle proteine animali trasformate nei mangimi, saggiando tre differenti protocolli, tratti dalla letteratura, verificando i limiti di rivelabilità (LOD) per le differenti specie animali in farine e mangimi contaminati con PAT, e verificandone l'applicabilità in ambito quantitativo.

\section{Materiali e Metodi}

\section{Selezione dei campioni ed estrazione del DNA}

Per l'allestimento delle prove in RT-PCR per la determinazione e quantificazione di costituenti di origine animale sono state considerate tre diverse tipologie di matrice: i) farine animali pure (ruminante, pollo, suino); ii) un mangime certificato dall'EURL contaminato con PAT bovino $0,1 \%$; iii) DNA genomico estratto da tessuto muscolare di bovino, pollo e suino.

L'estrazione del DNA è stata effettuata tramite il kit PureLink ${ }^{\circledR}$ Genomic DNA (Life Technologies Ltd, Paisley, UK), seguendo le indicazioni fornite dal produttore. La concentrazione di DNA totale estratto dai campioni in esame è stata determinata tramite fluorometro, utilizzando il kit Quant-iTTM dsDNA HS assay (Life Technologies Ltd).

A partire dai campioni di DNA genomico estratti da tessuto muscolare (tipologia 3) sono state preparate da cinque a otto diluizioni in base dieci per l'allestimento di curve standard.

\section{Saggi real time-polymerase chain reaction}

I saggi e i set primer-sonde applicati ai campioni estratti sono stati reperiti dalla bibliografia e riportati in Tabella 1.

Per ogni protocollo utilizzato sono stati applicati i parametri di corsa suggeriti dai rispettivi autori (concentrazioni di primer e probe, quantitativi di DNA caricato per reazione, profili termici di amplificazione). Le corse sono state eseguite su Step0neTM Plus (Life Technologies Ltd), utilizzando Universal Master Mix 2X (Life Technologies Ltd) e acqua UP DNAsi RNAsi free (Sigma Aldrich, St. Louis, M0, USA). Ogni campione è stato analizzato in triplicato.

Il protocollo 1, basato sul lavoro di LópezAndreo et al. (2005), utilizza primer e sonde
Correspondence: Beatrice Brusa, Istituto Zooprofilattico Sperimentale del Piemonte, Liguria e Valle D’Aosta, via Bologna 148, 10154 Torino, Italy.

Tel. +39.011.2686252 - Fax: +39.011.2686228.

E-mail: beatrice.brusa@izsto.it

Key words: Processed animal proteins, Quantification, polymerase chain reaction .

Conflict of interests: the authors declare no potential conflict of interests.

Received for publication: 15 January 2013.

Revision received: 25 February 2013.

Accepted for publication: 26 February 2013.

This work is licensed under a Creative Commons Attribution 3.0 License (by-nc 3.0)

(C) Copyright D. Marchis et al., 2013

Licensee PAGEPress, Italy

Italian Journal of Food Safety 2013; 2:e4

doi:10.4081/ijfs.2013.e4

selezionati a partire da target genetici sovrapponibili (regioni comuni del DNA mitocondriale), permettendo il disegno di saggi Taqman (5'FAM-3'MGB) che utilizzano probe comuni e coppie di primer differenti. Tale scelta è stata giustificata dagli autori come necessaria al contenimento dei costi che deriverebbero invece dall'uso di distinti set di primer probe. I primer specie-specifici utilizzati per la determinazione di bovino e suino sono disegnati su regioni variabili del DNA mitocondriale (gene per la t-Glu, gene ND5, cyt B).

I saggi descritti nel protocollo 2 da Krcmar e Rencova (2005) si basano su sequenze oligonucleotidiche disegnate su target specie-specifici localizzati sul DNA mitocondriale, (regione $t$ RNALys specifica per pollo, subunità 6 e 8 dell'ATPasi specifiche per bovino e suino). Le sonde Taqman utilizzate sono di tipo 5'FAM3'Black Hole 1.

Il protocollo 3, descritto da Mendoza-Romero et al. (2004), prevede l'utilizzo di un saggio di tipo Taqman (5'FAM-3'TAMRA) rivolto verso il target genetico nucleare specifico per ruminanti (il trasposone SINE Bov-A2), a differenza degli altri protocolli considerati, entrambi basati su target mitocondriali.

\section{Analisi dei dati e quantificazione}

Per l'analisi delle corse si è utilizzato il software in dotazione allo strumento (Step0ne software 2.2), tramite il quale sono stati determinati i parametri delle curve standard allestite sulle diluzioni degli estratti (slope, $\mathrm{R}^{2}$, intercetta, efficienza di reazione), ed i risultati quantitativi riferiti al campione di riferimento dell'EURL (a concentrazione nota dello 0,1\% $\mathrm{w} / \mathrm{w})$. 


\section{Risultati}

Le curve standard e i dati a partire da diluizioni di DNA estratto da muscolo di bovino, suino e pollo sono riassunte nella Tabella 2 . Un esempio di allestimento di curva standard su diluizioni seriali è mostrato nella Figura 1 .

\section{Protocollo 1}

Il LOD determinato per il saggio bovino calcolato considerando il $40^{\circ}$ ciclo di amplificazione come cut-off, è risultato $6.8 \mathrm{fg} / \mathrm{uL}$ (migliore rispetto a quello dichiarato nel lavoro originale a parità di cut-off). Tale situazione si è verificata anche per il saggio specifico per DNA suino $(0,01 \mathrm{pg} / \mathrm{uL}$ contro gli $0,07 \mathrm{pg} / \mathrm{uL}$ del paper originale).

\section{Protocollo 2}

Le curve standard costruite su diluizioni in base dieci dei controlli positivi utilizzati (DNA da PAT pure) hanno permesso di fissare i LOD del metodo per le varie specie. Per il saggio specifico per pollo e specie avicole il LOD è pari a $3,8 \mathrm{pg} / \mathrm{uL}$, per quello su suino è di 0,031 ng/uL e per il saggio specifico per bovino è pari a $0,05 \mathrm{ng} / \mathrm{uL}$.

\section{Protocollo 3}

Le prove effettuate a partire dalle diluizioni di DNA genomico, ripetute in triplicato, portano a definire un LOD su target ruminante di circa 2,5 fg/uL (cut-off $36 \mathrm{Ct}$ ).

Le conclusive analisi quantitative dei campioni di farine pure (100\% peso/peso) e del campione certificato dell'EURL (miscela 0,1\% bovino) sono riportate nella Tabella 3.

I valori quantitativi sugli estratti da farine sono stati determinati contro le rispettive curve standard a partire da estratti da tessuto muscolare per ciascuno dei 3 protocolli applicati.

\section{Discussione}

I valori di sensibilità analitica descritti dagli autori dei lavori presi in esame sono riferiti a quantitativi di target analitico (copie genomiche o concentrazione di DNA target nel campione). Per poter valutare la possibilità di tradurre questo dato quantitativo, riferito alle concentrazioni di DNA, in una valutazione quantitativa riferibile a concentrazioni peso/peso in miscele complesse come i mangimi, sono state determinate le performance di alcuni metodi reperiti in bibliografia basati sulla tecnologia RT-PCR.

Tutti i risultati ottenuti applicando i metodi considerati mettono in luce quanto il campione di riferimento fornito dall'EURL (0,1\% PAT bovino peso/peso) e le farine animali pure di bovino, suino e pollo siano quantificate diver-
Tabella 1. Primer e probe set dei tre protocolli impiegati.

\begin{tabular}{lllll} 
Protocollo & Target & Primer & Probe & Riferimenti \\
1 & Bovino & F8108/R8231 & P8135 & Krcmar e Rencova (2005) \\
& Pollo & F9188/R9277 & P9231 & \\
& Suino & F7975/R8064 & P8021 & \\
2 & Bovino & FBOS3/RBOS1 & S1 & López-Andreo et al. (2005) \\
& Pollo & FA1/RA1 & S7 & \\
& Suino & FC1SUS/RSUS2 & S1 & \\
\hline 3 & Ruminante & PRF/PRR & SINE & Mendoza-Romero et al. (2004) \\
\hline
\end{tabular}

Tabella 2. Curve standard allestite sulla base dei tre protocolli applicati.

\begin{tabular}{llllll}
$\begin{array}{l}\text { Caratteristiche curve standard } \\
\text { Protocollo }\end{array}$ & Slope & Efficienza & Intercetta & $\mathbb{R}^{2}$ \\
\multirow{2}{*}{$\begin{array}{l}\text { Specie } \\
\end{array}$} & Bovino & $-3,738$ & $85,14 \%$ & 20,69 & 0,999 \\
& Pollo & $-3,458$ & $94,62 \%$ & 23,47 & 0,999 \\
\multirow{2}{*}{2} & Suino & $-3,946$ & $79,22 \%$ & 20,11 & 0,999 \\
& Bovino & $-3,568$ & $90,66 \%$ & 19,32 & 0,998 \\
& Pollo & $-3,401$ & $96,81 \%$ & 26,52 & 0,998 \\
& Suino & $-3,661$ & $87,56 \%$ & 18,72 & 0,999 \\
\hline 3 & Ruminante & $-3,344$ & $99,08 \%$ & 15,09 & 0,996 \\
\hline
\end{tabular}

Tabella 3. Analisi quantitative dei campioni di farine pure (100\% peso/peso) e del campione certificato dell'EURL (miscela $0,1 \%$ bovino) tramite i tre protocolli real time-polymerase chain reaction presi in esame. I valori quantitativi sugli estratti da farine sono stati determinati contro le rispettive curve standard a partire da estratti da tessuto muscolare.

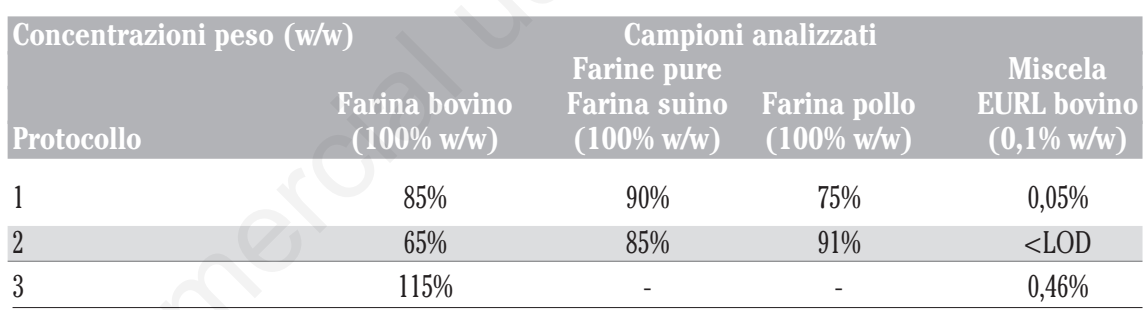

EURL, European reference laboratory; LOD, limite di rivelabilità.

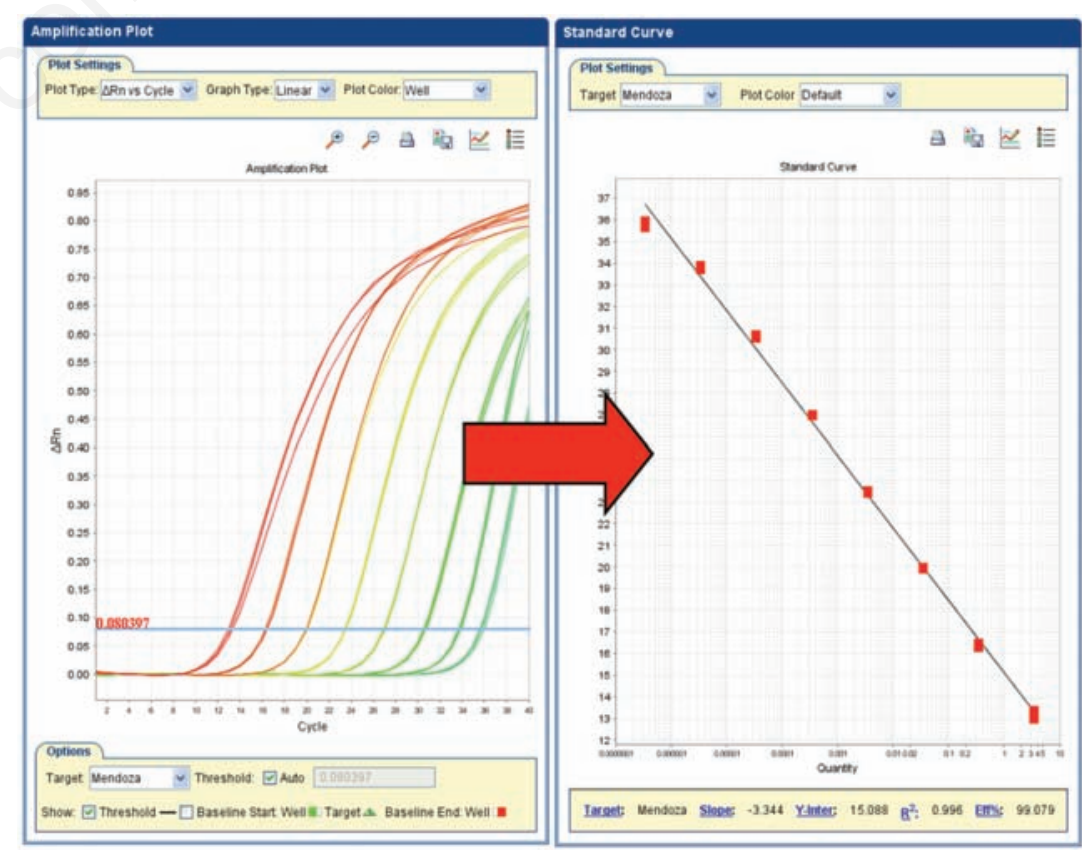

Figura 1. Profilo di amplificazione di una serie di diluizioni in base dieci (amplification plot) e relativa curva standard (standard curve). 
samente applicando curve standard ottenute dalle diluizioni seriali di DNA estratto da muscolo (Tabella 3). Tale incongruenza è ascrivibile a due limitazioni principali: i) natura dei target genetici utilizzati (target multicopia il cui numero può variare notevolmente a seconda della densità mitocondriale del campione in analisi 0 al numero di eventi di trasposizione/duplicazione del target ruminante all'interno del genoma); ii) limitazioni tecniche nell'estrazione del DNA: differenze nei recuperi, presenza di DNA degradato (quantificabile ma non amplificabile) a causa dei trattamenti termici a cui sono sottoposti in generale i mangimi).

Anche allestendo le curve standard a partire dagli estratti di PAT pure, cercando di limitare quindi l'effetto matrice, la quantificazione del campione di riferimento allo $0,1 \%(\mathrm{w} / \mathrm{w})$ rimane errata per tutti e tre i protocolli presi in esame (dati non mostrati), confermando come il limite di applicabilità del metodo quantitativo in RT-PCR sia dovuto alla scelta di target genetici a numero di copie variabili. Questa evidenza suggerisce come al momento non sia possibile, in mancanza delle singole materie prime (la/le singole farine contenenti PAT) utilizzate per la preparazione di mangimi, allestire curve standard appropriate su cui poter quantificare con precisione le concentrazioni peso/peso nei prodotti finiti (formulazioni basate su miscele delle singole materie prime, vegetali e contenenti PAT).

Similmente a quanto suggerito da alcuni autori (Benedetto et al., 2011), la scelta di target genetici nucleari, presenti in un numero di copie definito all'interno del genoma (2n copie in genomi diploidi) permetterebbe l'allestimento di metodiche quantitative sicuramente più precise, similmente a quanto viene fatto nelle analisi quantitative degli OGM in materie prime vegetali.

Tali metodiche risulterebbero sicuramente più precise di quelle allestibili utilizzando i target multicopia come quelli utilizzati in questo lavoro. Per contro, la scelta di target nucleari a numero di copie genomiche fisso limiterebbe molto la sensibilità analitica dei metodi derivati, questo principalmente a causa dei processi di trasformazione a cui sono sottoposti mangimi, pellet e pet-food (estrusione, tecniche molitorie e alte temperature di processazione che danneggiano e frammentano il DNA).

\section{Conclusioni}

L'analisi preliminare svolta in questo lavoro permetterà ulteriori approfondimenti necessari allo sviluppo di metodi quantitativi.

Tali metodiche risultano infatti direttamen- te applicabili nel monitoraggio di eventi di contaminazione, accidentali o fraudolenti, generati dalla reintroduzione delle PAT nell'alimentazione animale.

\section{Bibliografia}

Benedetto A, Abete MC, Squadrone S, 2011. Towards a quantitative application of Real Time PCR technique for fish DNA detection in feedstuffs. Food Chem 126:1436-42.

Commissione Europea, 2010. TSE roadmap II: a strategy paper on transmissible spongiform encephalopathies for 2010-2015. Commissione Europea ed., Bruxelles, Belgio.

Krcmar P, Rencova E, 2005. Quantitative detection of species-specific DNA in feedstuffs and fish meal. J Food Protect 68:1217-21.

López-Andreo M, Lugo L, Garrido-Pertierra A, Prieto MI, Puyet A, 2005. Identification and quantitation of species in complex DNA mixtures by real-time polymerase chain reaction. Anal Biochem 339:73-82.

Mendoza-Romero L, Verkaar EL, Savelkoul PH, Catsburg A, Aarts HJ, Buntjer JB, Lenstra JA, 2004. Real-time PCR detection of ruminant DNA. J Food Protect 67:550-4. 